\title{
Single Element Excitation and Detection of (Micro-)Mechanical Resonators
}

\author{
Harrie A.C. Tilmans, Dominicus J. IJntema and Jan H.J. Fluitman
}

MESA, Institute for Micro Electronics, Materials Engineering, Sensors \& Actuators, University of Twente, P.O. Box 217, 7500 AE Enschede, The Netherlands

\begin{abstract}
This paper describes a single-element approach for the excitation and detection of the vibrational motion of (micro-) mechanical resonators. An equivalent electrical one-port network is derived for an electrostatically and a piezoelectrically driven resonator. In this way, the effect of the mechanical resonator is transformed into the electrical domain and can easily be accounted for in a circuit simulation. Further, a detection circuit, based on an (on-chip) bridged design is proposed as a way to compensate for the parasitic parallel load of the one-port. A criterion is given for the accepted level of unbalance of the bridge if a minimal phase shift of $90^{\circ}$ in the transfer characteristic around resonance is demanded.
\end{abstract}

\section{Introduction}

In principle two approaches can be used for the excitation and detection of the vibrational motion of a mechanical resonator. One approach uses separate elements for excitation and detection of the vibration and can be modeled as an electrical two-port network $[1,2,3]$. The other approach uses a single element for both excitation and detection and can be modeled as an electrical one-port network $[4,5,6]$. In both approaches, spurious responses can obscure the mechanical resonance. For the two-port configuration this is caused by electrical feedthrough from the driving port to the detection port. For the one-port configuration electrical feedthrough (by definition) does not exist, since there is only one port. However, here a parasitic parallel load has a similar degrading effect as the electrical feedthrough in a two-port network. The choice for a specific approach depends on the choice of the transduction mechanism [7], on the complexity of the layout and fabrication process, on the electronic circuitry used for the oscillator, and also on the question whether the problem of the spurious response as mentioned above can adequately be solved or not.

An equivalent electrical one-port network of an electrostatically and a piezo-electrically driven resonator is derived. This equivalent network is subsequently implemented in the simulation of a detection circuit for the mechanical resonance.

\section{Theoretical Model}

The development of an equivalent one-port network of the resonator is based on a modal analysis [8]. A homogeneous, prismatic, wide beam $(b>5 h,[14])$ with a rectangular cross section and clamped at both ends, is taken as an example. Two different methods of excitation/detection of the vibrational motion are discussed (Fig. 1).

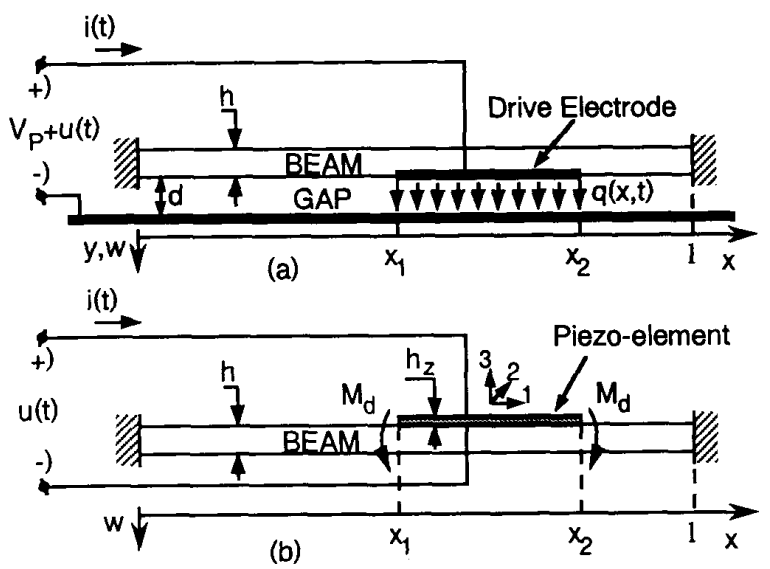

Fig. 1 Sketch of a resonating beam driven by means of a single element: (a) electrostatically excited and capacitively detected, (b) piezoelectrically excited and detected.

One method uses electrostatic excitation/detection (air-gap capacitor) $[2,3,6]$. Here the structure is excited by means of a transverse force. The other method uses piezo-electric excitation/detection [1], where the driving load is a bending moment. The differential equation of motion governing the deflection $w(x, t)$ of the beam, subjected to an axial (tensile) load $N$ and a transverse driving load $q(x, t)$, can be expressed as

$$
\frac{E I}{1-v^{2}} \frac{\partial^{4} w(x, t)}{\partial x^{4}}-N \frac{\partial^{2} w(x, t)}{\partial x^{2}}+\varrho b h \frac{\partial^{2} w(x, t)}{\partial t^{2}}+c \frac{\partial w(x, t)}{\partial t}=q(x, t)
$$

where $E, v, I, \varrho, c, b, h, x$ and $t$ are the Young's modulus, Poisson's ratio, second moment of inertia, specific mass, viscous drag parameter, width and thickness of the beam, position along the beam length and time, respectively. In the following, a harmonic driving load with radial frequency $\omega$ is assumed: $q(x, t)=q(x) \mathrm{e}^{\mathrm{j} \omega x}$. The steady state solution to eq. (1) may be expressed as an infinite series of the mode shape functions $\phi_{n}(x)[8]$ :

$w(x, t)=\sum_{n=1}^{\infty} B_{n} \phi_{n}(x) e^{j \omega t}$

Substituting eq. (2) into eq. (1) and using the orthogonality

91CH2817-5/91/0000-0533\$01.00 C) 1991IEEE 
properties of the mode shape functions, an expression for the coefficients $B_{n}$ is found, yielding

$w(x, t)=\sum_{n=1}^{\infty} \frac{P_{n} \phi_{n}(x) e^{j \omega t}}{\omega_{n}^{2}\left[1+\frac{1}{Q_{n}}\left(\frac{j \omega}{\omega_{n}}\right)+\left(\frac{j \omega}{\omega_{n}}\right)^{2}\right] M_{n}}$

where

$$
\begin{array}{ll}
P_{n}=\int_{0}^{l} q(x) \phi_{n}(x) d x & \text { (generalized load) } \\
M_{n}=\int_{0}^{l} \mathrm{\rho} b h \phi_{n}^{2}(x) d x & \text { (generalized mass) }
\end{array}
$$

and, $Q_{\mathrm{n}}=\left(\mathrm{Q} b h \omega_{\mathrm{n}}\right) / c$ the quality factor of the $\mathrm{n}$-th mode, $l$ is the beam length and $\omega_{n}$ the natural frequency of the $n$-th mode. The mode shape functions $\phi_{n}$ and the natural frequencies $\omega_{n}$ (see also eq. (15)) can be found by solving the eigenvalue problem associated with eq. (1) [14]. The generalized load $P_{\mathrm{n}}$ can be seen as a measure for the efficiency of excitation of a particular mode. It is obvious that a large value for $\boldsymbol{P}_{\mathrm{n}}$ results in a large contribution of mode $n$ to the overall response. To excite a symmetric mode with respect to the centre of the beam (e.g. the fundamental mode), the driving load $q(x)$ should also be symmetric. Asymmetric modes will be suppressed in this case. Theoretically it is even possible to excite the first mode (symmetric) and to suppress both the second (asymmetric) and the third mode (symmetric). Note that in this case the first mode will not be excited in the most efficient way.

\section{Piezo-electric Excitation/Detection}

To vibrate the structure depicted in Fig. 1(b), an ac drive voltage $u(t)=u \mathrm{e}^{\mathrm{j} \omega \mathrm{t}}$ is applied across the piezo-element. Due to the piezo-electric effect, an electric field perpendicular to the surface (direction " 3 ") induces an in-plane (directions "1" and "2") deformation of the element, which causes bending moments $M_{\mathrm{d}}$ to be exerted on the supporting beam. In case of a single piezo-element, e.g., made of $\mathrm{ZnO}$, extending from $x_{1}$ to $x_{2}$ as sketched in Fig. 1(b), the driving load $q(x)$ can be expressed as

$$
q(x)=-M_{d}\left[\delta_{-1}\left(x-x_{1}\right)-\delta_{-1}\left(x-x_{2}\right)\right]
$$

where $M_{\mathrm{d}}$ is the magnitude of the bending moment induced by the voltage $u$ applied across the piezo-element and $\delta_{-1}$ represents the unit doublet function (derivative of the Dirac function). The generalized load now becomes:

$$
P_{n}=\int_{0}^{l} q(x) \phi_{n}(x) d x=M_{d}\left[\phi_{n}^{\prime}\left(x_{1}\right)-\phi_{n}^{\prime}\left(x_{2}\right)\right]
$$

where $\phi_{\mathrm{n}}{ }^{\prime}$ represents the spatial derivative of the mode shape functions. The magnitude of the driving moment $M_{\mathrm{d}}$ depends on the material properties, dimensions and the applied voltage. Assuming the same Young's modulus for the beam material and the piezo-electric layer, and further a small thickness $h_{\mathrm{z}}$ of the piezo-element $\left(h_{z} \ll h\right), M_{\mathrm{d}}$ can be approximated by [9]

$$
M_{d}(t)=\frac{E h b d_{31}}{2(1-v)} u(t)=\frac{E h b d_{31}}{2(1-v)} u e^{j \alpha x}
$$

where $E$ and $\nu$ are Young's modulus and Poisson's ratio of both materials, and $d_{31}$ is the piezo-electric constant of the piezo-layer. The detection current $i(t)$ (Fig. 1(b)) can be expressed as

$$
i(t)=\iint_{A} \frac{\partial D_{3}(x, t)}{\partial t} d A=j \omega b \int_{x_{1}}^{x_{2}} D_{3}(x) d x
$$

where $D_{3}$ is the dielectric displacement and $A$ the total electrode area $\left(A=\left(x_{2}-x_{1}\right) b\right)$. An expression for $D_{3}$ can be found from the constitutive equations [10] and for $h_{z} \ll h$ it is given by (only including bending strains and ignoring the axial strain):

$D_{3}(x)=-d_{31} \frac{E}{1-v^{2}} \frac{h}{2} \frac{\partial^{2} w(x)}{\partial x^{2}}+\varepsilon_{33}^{T}\left(1-k_{31}^{2}\right) \frac{u}{h_{z}}$

where $\varepsilon_{33}{ }^{\mathrm{T}}$ and $k_{31}$ are the free permittivity and coupling coefficient of the piezo-material, respectively [10]. Combining eqns. (3), (5), (6), (7) and (8) yields the admittance $Y(j \omega)$ :

$$
\begin{aligned}
Y(j \omega) & \equiv \frac{i}{u}=j \omega b\left(x_{2}-x_{1}\right) \frac{\varepsilon_{33}^{T}\left(1-k_{31}^{2}\right)}{h_{z}} \\
& +\sum_{n=1}^{\infty} \frac{j \omega}{\left(1-v^{2}\right)(1-v)} \frac{\frac{1}{4} b^{2} h^{2} E^{2} d_{31}^{2}\left(\phi_{n}^{\prime}\left(x_{1}\right)-\phi_{n}^{\prime}\left(x_{2}\right)\right)^{2}}{\omega_{n}^{2}\left[1+\frac{1}{Q_{n}}\left(\frac{j \omega}{\omega_{n}}\right)+\left(\frac{j \omega}{\omega_{n}}\right)^{2}\right]_{n}}
\end{aligned}
$$

A more detailed analysis, including the effect of the piezoelement (with a different Young's modulus) on the stiffness and on the position of the neutral axis will be published elsewhere [11].

\section{Electrostatic Excitation/Detection}

The motional air-gap capacitor depicted in Fig. 1(a) is excited by applying an ac drive voltage $u(t)$ and a dc polarization voltage $V_{p}$ across the (air)gap [2,6,12]. Assuming the direction of the electric field vector to be perpendicular to the $x$-axis along the entire length of the beam, the ac driving load can be expressed as $\left(u \kappa V_{\mathrm{p}}\right)$

$$
q(x)=\frac{\varepsilon_{0} \varepsilon, p u V_{P}}{\left[d-y_{\text {stat }}(x)\right]^{2}}
$$

where $\varepsilon_{o}$ is the dielectric constant of vacuum, $\varepsilon_{\mathrm{r}}$ the permittivity of the gap medium, e.g., air, $d$ the zero-voltage gap spacing and $y_{\text {stu }}(x)$ the static deflection caused by $V_{\mathrm{p}}$. The small signal detection current $i(t)$ flowing into the port is given by $\left(u \ll V_{\mathrm{P}}, w(x) \ll\left[d-y_{\text {sta }}(x)\right]\right)$

$$
i(t)=C_{0} \frac{d u}{d t}+V_{P} \frac{d C}{d t}
$$

where

$$
\begin{aligned}
& C_{0}=\varepsilon_{0} \varepsilon_{r} b \int_{x_{1}}^{x_{2}} \frac{d x}{d-y_{\text {stat }}(x)} \quad \text { (static capacitance) } \\
& \frac{d C}{d t}=\varepsilon_{0} \varepsilon_{r} b \int_{x_{1}}^{x_{2}} \frac{j \omega w(x)}{\left[d-y_{\text {stat }}(x)\right]^{2}} d x
\end{aligned}
$$

and $w(x)$ is the amplitude of the vibration. The second term in eq. (11) is due to the motion of the resonator. Combining eqns. 
(3), (10), (11) and (13) yields an expression for the admittance $Y(j \omega)$ of the electrostatic one-port network:

$$
\begin{aligned}
& Y(j \omega) \equiv \frac{i}{u}=j \omega C_{o}+
\end{aligned}
$$

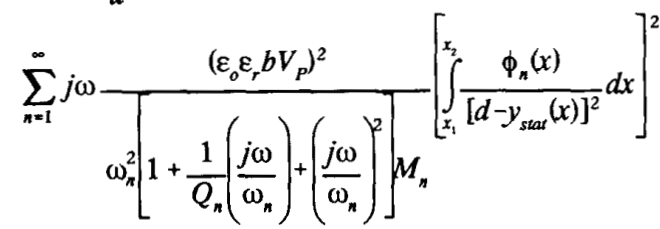

The attractive force caused by the polarization voltage $V_{p}$, is a function of the deflection of the beam. An increase of the deflection results in a decrease of the gap spacing and thus in an increase of the electrostatic force. If $V_{\mathrm{P}}$ exceeds the so called pull-in voltage $V_{\mathrm{Pr}}$, the system becomes mechanically instable and the gap spacing immediately goes to zero [12]. The static deflection curve $y_{\text {stat }}(x)$ can be found by numerically solving the differential equation (1), thereby setting the time derivatives equal to zero. Subsequently, the pull-in voltage is determined by a numerical iterative procedure. Details of the static behaviour will not be given here, but will be published elsewhere [13]. Another effect of the polarization voltage is the lowering of the natural frequency of the structure due to the gradient in the transverse (electrostatic) force, which varies with $V_{\mathrm{p}}$ [12]. A good approximation for the resonant frequency can be found from an analysis based on Rayleigh's energy method [8]. Besides the contributions to the potential energy due to bending and axial deformation of the beam an additional term, representing the change in energy of the electric field in the gap due to the vibration must be included:

$$
\omega^{2}=\frac{\frac{E I}{1-v^{2}} \int_{0}^{l}\left(\frac{d^{2} \tilde{w}}{d x^{2}}\right)^{2} d x+N \int_{0}^{l}\left(\frac{d \tilde{w}}{d x}\right)^{2} d x-\int_{x_{1}}^{x_{2}} \frac{\varepsilon_{0} \varepsilon b V_{P}^{2} \tilde{w}^{2}(x)}{\left[d-y_{\text {stat }}(x)\right]^{3}} d x}{\operatorname{\rho bh} \int_{0}^{l} \tilde{w}^{2}(x) d x}
$$

where $\tilde{w}(x)$ is the assumed shape of the particular mode. In the following examples, the mode shape for zero axial strain is used. At the pull-in voltage, the fundamental frequency equals zero. This behaviour is similar to the buckling phenomenon [14]. In both cases, the system becomes mechanically instable and the resonant frequency approaches zero. Because of the complexity of the air-gap capacitor, which makes it not suitable to do quick design calculations ("engineering approach"), it would be attractive to have a simple model which predicts the behaviour of the actual system around the first mode with sufficient accuracy. A lumped spring-mass system provides such a model $[6,12]$. The zero-voltage gap spacing $d$ and the electrode area $A=\left(x_{2}-x_{1}\right) b$ are chosen to be the same for both systems. The spring constant $K_{\mathrm{o}}$ of the spring-mass system is simply the static constant seen at the centre of the beam. For a clampedclamped beam and with the driving electrode extending along the entire beam length, $K_{\mathrm{o}}$ is given by: $K_{\mathrm{o}}=384 E I /\left[\left(1-v^{2}\right) l^{3}\right]$. The spring constant can be corrected to include the effect of axial strain $\varepsilon$ by adding an additional term $K_{\mathrm{e}}=K_{\mathrm{o}} 0.295 \varepsilon\left(1-v^{2}\right)(l / h)^{2}$. Thus, the overall spring constant is given by: $K=K_{\mathrm{o}}+K_{e}$. The equivalent mass is found by setting the resonant frequencies of both systems equal to each other at $V_{\mathrm{P}}=0: M=K / \omega_{1}$. An expression for the pull-in voltage $V_{P I}^{s m}$ of the spring-mass system can easily be derived and is given by [12]:

$V_{P I}^{s m}=\sqrt{\frac{8}{27} \frac{K d^{3}}{\varepsilon_{o} \varepsilon_{r} A}}$

It turns out, that the estimated pull-in voltage $V_{P T}^{s m}$ is roughly $10 \%$ lower than the "exact" value computed for the continuous beam. The estimated modal capacitance $C_{1}$ of the spring-mass system is roughly a factor 1.5 too large. The resonant frequencies as a function of the polarization voltage are approximately equal. Examples comparing the results obtained from the spring-mass model to the model of the continuous beam are given in Fig. 2. A more detailed discussion will be published elsewhere [13].

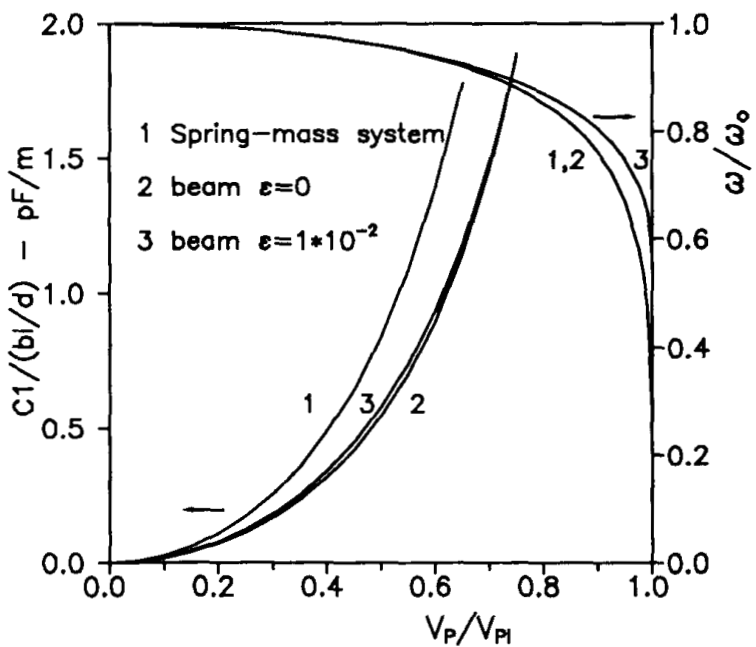

Fig. 2 The normalized modal capacitance and the normalized angular resonant frequency versus the normalized polarization voltage of an electrostatically driven beam. Curves 1 and 2 of the angular resonant frequency approximately coincide. $\omega_{0}$ is the resonant frequency at $V_{P}=0$.

\section{Equivalent Electrical One-Port Network}

Both the piezo-electrically and electrostatically driven resonator can be modeled by the same electrical one-port network. This is obvious by a comparison of eqns. (9) and (14). The equivalent network is shown in Fig. 3, and the component values are given in Table $\mathrm{I}$. By modeling the mechanical resonator as an electrical network, the resonator behaviour can easily be accounted for in a circuit simulation, e.g., SPICE.

\section{Detection of the Resonance}

The resonant frequency of the resonator can be found from the admittance plot of the one-port network. A parasitic load, e.g., a parallel capacitor $C_{\mathrm{p}}$, can obscure the resonance. A criterion for proper detection of the first mode would be: $Q_{1} C_{1} 》\left(C_{\mathrm{p}}+C_{\mathrm{d}}\right)$. If this criterion is satisfied the resonator can be used in a standard (quartz) crystal oscillator. Typical values for $C_{\mathrm{p}}+C_{\mathrm{o}}$ 


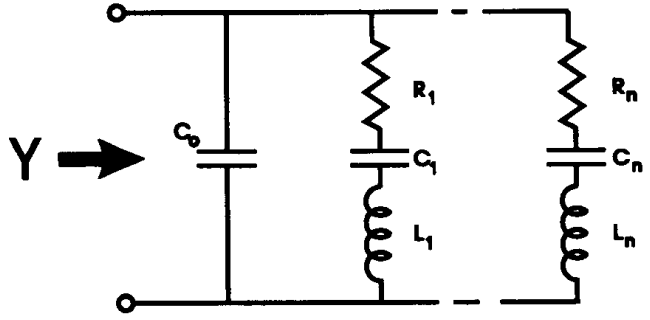

Fig. 3 Equivalent electrical network of the piezo-electrically and electrostatically driven resonator.

Table I. Static and motional capacitance values of the network of Fig. 3. The inductance is given by $L_{n}=1 /\left(\omega_{n}{ }^{2} C_{n}\right)$ and the resistance by $R_{n}=\left(11 Q_{n}\right) \sqrt{ }\left(L_{n} / C_{n}\right)$. The radial frequency $\omega_{n}$ can be approximated by eq. (15).

\begin{tabular}{|l|c|c|}
\cline { 2 - 3 } & $\mathrm{C}_{0}$ & $\mathrm{C}_{\mathrm{n}}$ \\
\hline $\begin{array}{l}\text { Electro- } \\
\text { static }\end{array}$ & $\varepsilon_{o} \varepsilon, b \int_{x_{1}}^{x_{2}} \frac{d x}{d-y_{\text {seat }}(x)}$ & $\frac{\left(\varepsilon_{o} \varepsilon, b V_{p}\right)^{2}}{\omega_{n}^{2} M_{n}}\left[\int_{x_{1}}^{x_{2}} \frac{\phi_{n}(x) d x}{\left[d-y_{\text {stat }}(x)\right]^{2}}\right]^{2}$ \\
\hline $\begin{array}{l}\text { Piezo- } \\
\text { electric }\end{array}$ & $b\left(x_{2}-x_{1}\right) \frac{\varepsilon_{33}^{T}\left(1-k_{31}^{2}\right)}{h_{z}}$ & $\frac{\frac{1}{4} b^{2} h^{2} E^{2} d_{31}^{2}\left(\phi_{n}^{\prime}\left(x_{1}\right)-\phi_{n}^{\prime}\left(x_{2}\right)\right)^{2}}{\left(1-v^{2}\right)(1-v) \omega_{n}^{2} M_{n}}$ \\
\hline
\end{tabular}

and $C_{1}$ of a resonator are on the order of $\mathrm{pF}$ 's and $\mathrm{fF}$ 's, respectively. This means that a $Q$-factor on the order of 10,000 is required. For resonators operating in a vacuum, this is achievable [15]. If the condition is not or marginally satisfied, a modified oscillator circuit is required. For instance, the resonator could be part of a (capacitive) divider circuit $[3,6]$. A method to largely eliminate the problem of the parallel load, e.g., a parallel capacitor, is based on a bridged design (see Fig. 4). Here, as opposed to the methods indicated above, compensation techniques are used. One (or more) branches of the bridge are formed by the resonator and the remaining branches are formed by one-port networks with an immittance equal to the immittance of the resonator far away from resonance. Note that the resulting network is modeled as a two-port network even though a single element is used for excitation/detection purposes. If a phase shift of roughly $90^{\circ}$ is demanded, the following approximate $\left(Q » 1, C_{1} \ll C_{\mathrm{o}}\right)$ condition can be derived for the bridge shown in Fig. 4:

$$
\frac{1}{\sqrt{1+\left(\frac{Q_{1} C_{1}}{C_{p}+C_{o}}\right)^{2}}}<\left(\frac{C_{p}+C_{o}}{C_{p 1}} \frac{C_{p 2}}{C_{p 3}}\right)<\sqrt{1+\left(\frac{Q_{1} C_{1}}{C_{p}+C_{o}}\right)^{2}}
$$

If the bridge is perfectly balanced, the term in the middle equals one and the above condition will always be satisfied. If a resonator is used with a static capacitance $C_{\mathrm{o}}+C_{\mathrm{p}}=1 \mathrm{pF}$, a

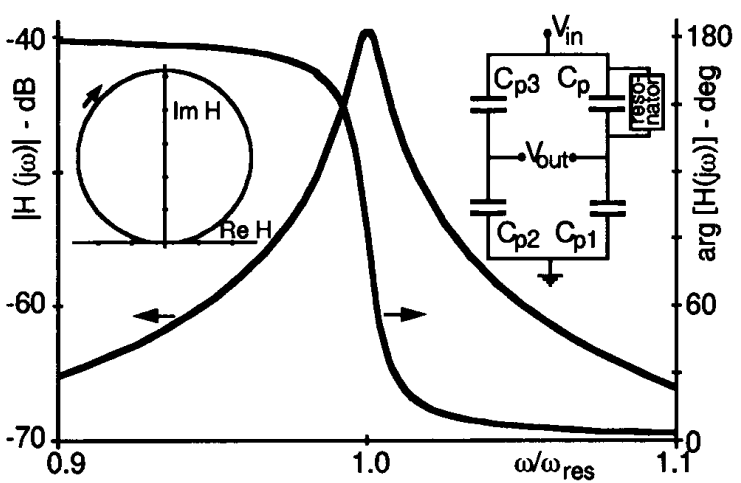

Fig. 4 Simulated Bode plot and polar diagram of the transfer function of the bridge used as a detection circuit for the resonating beam. The resonator is modeled by the network shown in Fig. 3 , thereby only including the first mode (or $R_{1} L_{1} C_{1}$-branch). $\omega_{\text {res }}$ denotes the resonant frequency of the first mode.

modal capacitance $C_{1}=10 \mathrm{fF}$ and a $Q_{1}=100$, an unbalance of $30-40 \%$ of the bridge can (theoretically) be accepted. Note that in this case, the admittance condition given at the beginning of this section is not satisfied. Or, in other words, if the admittance condition cannot be satisfied, it would still be possible to detect the resonance if a balanced bridged design is employed. Another relevant consideration is the noise or interference level. Larger values of the parasitic capacitances will result in a lowering of the peak value of the magnitude of the transfer function and eventually, the resonator signal cannot be distinguished anymore.

The insert in Fig. 4 shows a polar plot of the transfer function. In order to satisfy the Nyquist criterion to form a feedback oscillator, the polar plot should be rotated by $90^{\circ}$ (assuming a real positive gain). In an oscillator this can be done by including an integrator, introducing the $90^{\circ}$ in the loop. This concept has been successfully tested with piezo-electrically driven macroscopic models. The fabrication of micromechanical polysilicon resonators is now in progress. On-chip bridged designs are used, where the inactive branches are also formed by mechanical resonators, but with a resonant frequency which is different from the frequency of the active branches. This way, a proper balance of the bridge is achieved.

\section{Conclusions}

Theoretically it is shown that both the piezo-electrically and electrostatically driven resonator can be modeled by the same electrical one-port network. The influence of the parasitic parallel load can be compensated for by employing a bridged design. The accepted level of unbalance of the bridge depends on the quality factor and on the modal capacitor of the resonator and further on the parasitic capacitance(s). For engineering purposes, the electrostatically driven resonator can sufficiently accurate be described by a lumped spring-mass system. 


\section{Acknowledgements}

The authors would like to thank dr. Miko Elwenspoek, dr. Siebe Bouwstra and dr. Theo Lammerink for helpful discussions and suggestions.

\section{References}

[1] J.G. Smits, H.A.C. Tilmans and T.S.J. Lammerink, "Pressure dependence of resonant diaphragm pressure sensor", in Tech. Digest, Proc. 3th Int. Conf. Solid-State Sensors and Actuators (Transducers '85), Philadelphia, USA, June 1985, pp. 93-96.

[2] R.T. Howe and R.S. Muller, "Resonant-microbridge vapor sensor", IEEE Trans. on El. Dev., vol. ED-33, no. 4, pp. 499-506, 1986.

[3] C. Linder, E. Zimmerman and N.F. de Rooij, "Capacitive polysilicon resonator with MOS detection circuit", presented at EUROSENSORS IV, Karlsruhe, Germany, Oct. 1-3, 1990.

[4] P.R. Wyman, "A new force to frequency transducer", Digital Instrumentation, IEE Conf. Publ. 106, 1973, pp. 117-123.

[5] M.B. Othman and A. Brunnschweiler, "Electrothermally excited silicon beam mechanical resonator", Electronic Letters, vol. 23, no.14, pp. 728-730, 1987.

[6] M.W. Putty, S.C. Chang, R.T. Howe, A.L. Robinson and K.D. Wise, "One-port active polysilicon resonant microstructures", in Proc. IEEE Micro Electro Mechanical Systems Workshop, Salt Lake City, UT, U.S.A., Feb. 1989, pp. 60-65.
[7] M. Elwenspoek, F.R. Blom, S. Bouwstra, T.S.J. Lammerink, F.C.M. van de Pol, H.A.C. Tilmans, Th.J.A. Popma and J.H.J. Fluitman, "Transduction mechanisms and their applications in micromechanical devices", in Proc. IEEE MEMS' '89, 1989, pp. 126-132.

[8] L. Meirovitch, Elements of vibration analysis. New York: McGraw-Hill, 1975, ch. 5, pp. 190-231.

[9] J.J.L.M. van Vlerken, S. Bouwstra, F.R. Blom, J.H.J. Fluitman and P.C. Breedveld, "Finite-mode bondgraph model of a resonant silicon-beam force sensor", accepted for publication (Feb. 1991) in Int. J. Modeling and Simulation.

[10] D.A. Berlincourt, D.R. Curren and H. Jaffe, "Piezoelectric and piezomagnetic materials and their function in transducers", in W.P. Mason (ed.): Physical acoustics vol. IA, New York: Academic Press, 1964, pp.169-270.

[11] H.A.C. Tilmans et al., to be published.

[12] H.C. Nathanson, W.E. Newell, R.A. Wickstrom and J.R. Davis jr., "The resonant gate transistor", IEEE Trans. on El. Dev., vol. ED-14, no. 3, pp. 117-133, 1967.

[13] D.J. IJntema and H.A.C. Tilmans, "The air-gap capacitor", to be published.

[14] S. Bouwstra and H.J.M. Geijselaers, "On the resonance frequencies of microbridges", accepted for presentation at Transducers '91, San Francisco, USA, June 1991.

[15] T.V. Roszhart, "The effect of thermoelastic internal friction on the $\mathrm{Q}$ of micromachined silicon resonators", in Proc. IEEE Solid-State Sensor and Actuator Workshop, Hilton Head Island, SC, USA, June 4-7, 1990 , pp. 13-16. 